\title{
Penetrating Liver Injuries
}

\author{
Thomas M. Scalea
}

Published online: 16 April 2015

(C) Springer International Publishing AG 2015

\begin{abstract}
Injuries to the liver are common. In the past, virtually, all penetrating injuries to the liver were managed by laparotomy, direct surgical exploration of the injured liver and repair. However, stable patients with isolated liver injuries, particularly those with low grade injuries, are now able to be managed non-operatively. Operative management can be as simple as packing, suture repair, and/or application of topical hemostatics. More complicated repairs such as resectional debridement, finger fracture with direct ligation of bleeding vessels, and major non-anatomic liver resections are sometimes needed to obtain hemostasis in major liver injuries. Damage control surgical techniques seem ideally suited for major liver injuries. Hemostasis is obtained with packing and whatever other techniques are necessary. This is used as a bridge to angiographic embolization, which can be followed by unpacking and other operative therapy as needed when the patient is more stable. Minimally invasive techniques, such as laparoscopy, do not have a major role in the management of penetrating liver injuries, though that may be expanded as time goes on. Newer adjuncts for hemostasis, such as higher quality topical hemostatic bandages, as well as other innovative therapies, may soon become available. Hepatic failure is infrequent, even with major liver injury, but extracorporeal techniques can be used in the few cases where profound hepatic failure does occur.
\end{abstract}

This article is part of the Topical Collection on Penetrating Injuries to Solid Abdominal Viscera

T. M. Scalea $(\bowtie)$

Department of Surgery, R Adams Cowley Shock Trauma Center,

University of Maryland School of Medicine, 22 South Greene Street,

Baltimore, MD 21201, USA

e-mail: tscalea@umm.edu
Keywords Liver injury $\cdot$ Selective nonoperative management · Damage control · Angiographic embolization · Innovative hemostatics

\section{Introduction}

Injuries to the liver are common. The liver is the largest organ in the abdominal cavity. While it is somewhat protected by the rib cage on the right hand side, its size still makes it vulnerable to injury.

Management of liver injuries has drastically changed over the last 30 years. Diagnostic refinements such as CT scanning have replaced the more archaic diagnostics such as diagnostic peritoneal lavage (DPL). Nonoperative management of blunt liver injury has become the standard, and most liver injuries in stable patients can now be observed regardless of the grade. Some of the lessons learned for nonoperative management of blunt liver injuries have successfully been utilized in patients with penetrating liver injuries.

In an era where nonoperative management is so successful, operative experience for most trainees doing big liver cases has dropped dramatically. Thus, many surgeons finish training with a paucity of expertise concerning operative management of the liver, particularly following injury and in patients in shock. Thus, the surgeon may be called on to do a gunshot wound to the liver with significant hemorrhage and be illprepared to manage that patient.

In this article, I will review recent advances in the management of penetrating liver injures, reflecting on 30 years of practice in two of the largest volume trauma centers in the USA. Some information can be gleamed from data on blunt liver injuries and I will attempt to utilize that to illustrate important points of penetrating liver injuries. 


\section{Defining a Magnitude of the Problem}

Penetrating trauma has generally been decreasing in most major American cities. Whether it be better law enforcement, trauma prevention programs, or better education, penetrating trauma is decreased in most trauma centers. In one recent review from San Paulo, Brazil, penetrating trauma decreased from $75 \%$ of admissions in 2000 to $20 \%$ in 2010 [1]. Thus, the opportunity for surgeons in training to learn and master the skills necessary to treat serious penetrating liver injury is compromised.

Certainly, some expertise can be gained from doing a number of elective liver cases. The exposure for elective hepatic cases is similar to the exposure needed to manage serious liver injuries. Clearly, the surgical anatomy is the same as well. However, elective liver cases are still relatively rare and are sought after by surgical trainees. Thus, it is a real possibility that a finishing surgical chief resident will have done very few major liver cases when graduating.

There are some courses that exist that attempt to address this deficiency. Perhaps, the best of these is the Advanced Trauma Operative Management (ATOM) Course, popularized by Dr. Lenworth Jacobs and the group at Harford, now an American College Surgeon's Committee on Trauma Course [2]. ATOM utilizes a large swine model and is geared towards teaching the students management of penetrating injury. Unfortunately, the liver anatomy in swine is not at all similar to that of a human. Thus, while very helpful, one must question the applicability of the lessons learned in ATOM when specifically discussing hepatic injuries.

Simulated learning, such as utilizing computer programs would be another possibility. Unfortunately, at least at the time of this writing, simulation for major abdominal operations has not yet reached a level of sophistication that makes it useful. Laparoscopic skills can be taught via simulation, and thus, many surgically focused simulation centers stress procedures that can be done via laparoscopically. While open procedures will likely catch up in the future, we have much to learn before this is a reality.

Certainly, some expertise can be gleamed from discussion. At our weekly Fellows' Conference, I make it a point to go over step by step management of severe liver injuries at least four to five times each year. When a fellow finishes our program, they at least have a framework for approaching a serious liver injury. While this is clearly not as good as direct operative experience, knowing the steps and having a check list in one's mind can be very helpful when one encounters a serious liver injury.

What then should a young surgeon do when confronted with a grade IV injury to the right hepatic lobe after a gunshot wound? Rapid decision-making is critical. I remind our trainees that this is the perfect scenario to call in a senior partner who has more experience with liver injuries. That call should be made early because if the patient is in profound hemorrhagic shock or in extremis, even years of experience will not be sufficient to salvage the patient.

\section{Evaluation of the Patient With Liver Injury}

Many times, the diagnosis of penetrating liver injuries is made at the time of operative exploration. Anyone with a trajectory through the right upper quadrant will likely have injury to the liver. The wise surgeon will recognize this and at least review surgical options on the way to the operating room.

In the past, anyone with suspected transabdominal trajectory simply underwent diagnostic exploratory laparotomy [3]. Traditional surgical thought was that the risk of injury was sufficient and a number of either truly negative or nontherapeutic laparotomies were acceptable to mandate diagnostic exploration. Nonoperative management of selected patients with penetrating abdominal injury is now accepted. Those in shock and those with evisceration or peritonitis should undergo immediate abdominal exploration. A recent practice management guideline for selective nonoperative management of penetrating abdominal trauma from EAST suggest that laparotomy is not mandatory for stab wounds without defined tenderness or peritonitis or diffuse abdominal tenderness gunshot wound of the wounds are tangential and there are not signs of peritonitis $[4 \cdot \bullet]$.

In 1986, our group from King's County first documented the utility of CT scanning in stable patients with penetrating injury to the back and flank [5]. We utilized CT scan for its three-dimensional perspective, recognizing that the retroperitoneum, an area that could not be evaluated with DPL, and in which injuries may be clinically occult for hours or days, could be adequately evaluated with use of the CT scanner. Oral, intravenous, and rectal contrasts were given to delineate the important structures in the retroperitoneum. As experience increased, selective operative management was introduced for patients with isolated solid organ injury.

Nonoperative management of liver injuries was first reported by Demetriades et al. in 1986 [6]. The authors studied 21 patients with penetrating injury in the right upper quadrant. Liver injury was assumed based on trajectory. Patients were managed with serial exams and transfusions as needed. All of these patients were successfully observed.

Since that original description, nonoperative management of penetrating injuries to the liver has become an accepted strategy. There have been a number of reports, but most have had a relatively small number of patients [7-10]. All of these have stressed serial physical exams. Several have also detailed the use of angiographic embolization with patients having evidence of intraparenchymal vascular injury $[10,11]$. In the last few years, there have been several larger series demonstrating nonoperative management as a successful strategy. In 
a recent series from Egypt, 62 patients with penetrating liver injuries were evaluated. Five patients in shock required laparotomy. All others were successfully treated with observation [12]. In another series from Belo Horizonte, Brazil, 115 patients with gunshot wounds to the right thoracoabdomen were studied. Of these, 109 had liver injuries. Only four patients (3.5\%) failed observation [13]. Finally, in a series from South Africa, 63 patients with liver gunshot wound were observed [14]. There were 14 associated kidney injuries and one spleen injury. Only five patients (7\%) failed observation.

My own thoughts are that nonoperative management of penetrating liver injuries is safe when the patient clearly has an isolated injury to the liver. Right-sided diaphragmatic injuries are likely fine to observe, even in patients with gunshot wounds. Most civilian gunshot or stab wound diaphragm injuries will heal without problem. It is more complicated when the trajectory may potentially involve structures other than the liver. Patients with penetrating liver injuries with free fluid in Morrison's Pouch and/or in the right colonic gutter may well have bled from the liver injury but may also have concomitant injury to the colon, head of the pancreas, or duodenum. It is not clear if $\mathrm{CT}$ is able to differentiate these injuries from a person with only a liver injury. In those cases, I am likely to explore the patient because the consequences of missed injury to the duodenum or liver can be devastating. Those injuries, when fixed expeditiously should do quite well. However, a missed colon injury with peritonitis almost certainly mandates creation of a stoma, a significantly worse outcome than primary repair.

\section{Operative Management of Penetrating Liver Injuries}

The operative techniques for managing liver injuries have not changed substantially in the last 30 years. In 1986, Feliciano et al. reported on 1,000 consecutive cases of hepatic trauma [15]. The vast majority ( $86.4 \%$ ) sustained penetrating trauma. A number of these patients had relatively simple repair. In the modern day, they may well have been observed. Eight-two percent were treated by simple repair, defined by suture hepatorrhaphy (64\%) or drainage only (20\%). Approximately $4 \%$ had no repair at all or application of a simple topical agent. Those who required more complicated repair were treated with hepatorrhaphy, resectional debridement, or resection and packing. This accounted for only $12 \%$ of the total patients.

In a review by the group in Cali, Columbia in 2013, 538 patients with penetrating thoracoabdominal injury, were treated over 8 years [16*•]. One hundred forty-six patients had penetrating liver injuries. The authors, a very experienced group, suggest a five-step algorithm for management of penetrating injuries to the liver. Those with non-active surgical bleeding are treated with compression, topical hemostatics, or simple repair. Those with active bleeding are first treated with the Pringle maneuver and packing. Those who fail undergo finger fracture hepatotomy and vascular ligation. Only eight patients $(5 \%)$ in that series had injuries to the retrohepatic vena cava, portal vein, or suprahepatic cava. These patients were treated with a variety of techniques.

Patients that required the most complex injuries had much more blood in their abdomen ( $2.5 \mathrm{vs} 1.5 \mathrm{l})$ and a higher rate of associated injuries. These results are very similar to those reported by Feliciano et al. in 1986.

My own approach to the management of penetrating injuries to the liver is very similar to that of our Colombian colleagues. Perihepatic packing is a surgical technique that requires some degree of skill. Simply placing a number of laparotomy pads in the right upper quadrant is not sufficient. When using packing for even temporary hemostasis, packs should be placed above the liver and both medially and laterally. I then place a number of lap pads under the liver and tamponade hemorrhage by pushing the liver up into the right diaphragm.

If packing is sufficient, one should consider either leaving the patient packed or examining the wounds more fully. Clearly, injuries to the posterior portion of the liver are much harder to control than a superficial injury, particularly if it is in the left lobe. The Pringle maneuver can be helpful, but most liver injuries that require operative therapy have a hepatic vein component. Thus, the Pringle maneuver is rarely completely hemostatic.

Unlike blunt trauma where the zone of injury may be very wide, the zone of injury after penetrating injury is defined by the missile tract. Thus, hepatotomy or tractotomy is very attractive. I generally divide the uninjured liver, using serial applications of the Universal stapler. Other alternatives are to use finger fracture or cautery set on a high setting. This allows the surgeon to directly visualize the bleeding blood vessels and ligate them.

For deeper injuries, packing as a bridge to angiographic embolization or resectional debridement are both attractive options. When using resectional debridement, I apply Pringle maneuver and then direct pressure medial to the area of injury. I resect the liver using the Universal staplers. The hepatic vein is surprisingly constant in its anatomic location. If identified, it can be ligated with the suture. As pressure on the liver parenchyma is released, it is usually relatively easy to identify and ligate any branches that remain. As the Pringle maneuver is released, the larger branches of the hepatic artery and portal vein can be identified and ligated.

Another useful adjunct in the management of deep penetrating injuries, particularly to the right lobe of the liver, is balloon tamponade. The original description involved the use of a red rubber catheter and a Penrose drain [17]. The catheter with the Penrose drain attached is inserted into the track of penetrating liver injury. The balloon effect is created 
when the catheter is injected and the Penrose drain balloons up with sufficient pressure to tamponade relatively small arterial bleeding and venous bleeding. Recently, the group from Campinas, Brazil reported on 46 patients in which a SengstakenBlakemore balloon was used for penetrating injuries to the liver [18•]. In the 46 patients, effective hemorrhage control was achieved in all. Fourteen patients required re-intervention, but of those, only three had the balloon removed. The balloon was deflated for approximately three days post-op and was removed several days later. There is one other recent case report using this technique [19]. In addition, there was a recent case report of a stab wound to the right lobe of the liver that was cared for with balloon tamponade using a Penrose drain, a Foley catheter, and several silk ties [20]. The patient was then treated with hepatic vein stenting and recovered.

I find balloon tamponade to be successful, but use it very rarely. I prefer to use tractotomy with the stapling device as described above. For very deep injuries in the right lobe of the liver, packing as a bridge to angiographic embolization is my preferred method. I generally use balloon tamponade once every 12 to 24 months.

\section{Minimally Invasive Therapy}

The gold standard for care of penetrating liver injuries is laparotomy. This allows for inspection of the liver injury and therapy as discussed above. Laparoscopy however may have a role in the management of patients with penetrating liver injury. In a recent review on the role of laparoscopy in penetrating abdominal trauma, liver injuries represented a small percentage of the injuries diagnosed [21]. Clearly, laparoscopy has been used for some time, particularly in left-sided thoracoabdominal penetrating injuries to identify the possibility of diaphragm injury and then to repair the diaphragm if injured. However, many are concerned about the ability of even talented laparoscopists to be able to run the entirety of the small bowel and identify subtle injuries. Thus, exploratory laparoscopy is rarely used. In patients with isolated hepatic injury, laparoscopy can be used to identify the magnitude of injury, at least on the exterior surface of the liver. Hemoperitoneum can be evacuated and drains placed if necessary. This may extend the role of observation in patients with penetrating injuries to the liver.

We recently cared for a patient who presented with a rightsided thoracoabdominal stab wound and a grade IV liver injury (Fig. 1). He had angiographic embolization for a large pseudoaneurysm (Figs. 2 and 3). The patient developed bile leak up through his diaphragmatic defect and ultimately developed a biliary pulmonary fistula and he began coughing up bile (Fig. 4). ERCP failed to control the bile leak and the patient was taken to the operating room. He underwent a VATS and decortication, followed by laparoscopic repair of

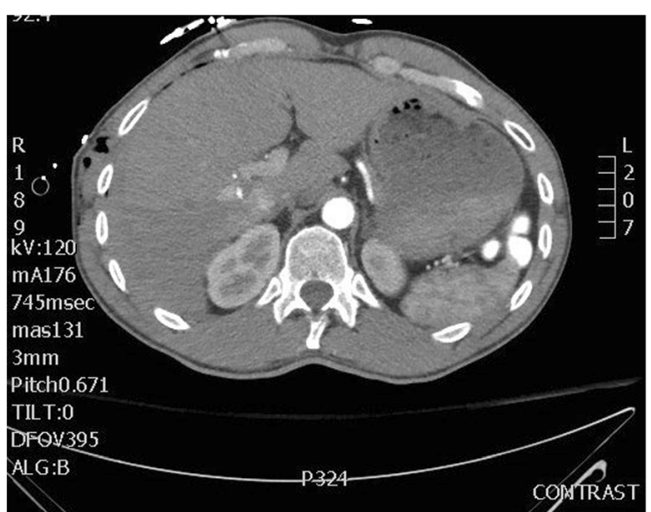

Fig. 1 A young man presented with a right-sided thoracoabdominal stab wound. CT demonstrated a grade IV liver injury with a vascular blush

his diaphragm, and placement of drains that successfully controlled the bile leak.

\section{Angiographic Embolization}

Angiographic embolization has been used to control hemorrhage from liver injuries for some time. This is most commonly used as part of damage control. Patients undergo operative exploration and control of hemorrhage as possible. The patients are packed and angiographic embolization was used to control bleeding deep in the liver parenchyma. Angiography sees the inside of the liver quite well and allows for placement of precise occlusion of injured blood vessels. Operative therapy for these deep vascular injuries would involve dividing a large volume of hepatic tissue, which may by itself cause significant hemorrhage.

Less well described is the use of angiographic embolization to extend nonoperative management of penetrating liver injuries. In a recent review, major hepatic necrosis was the most common complication of angiographic embolization of the

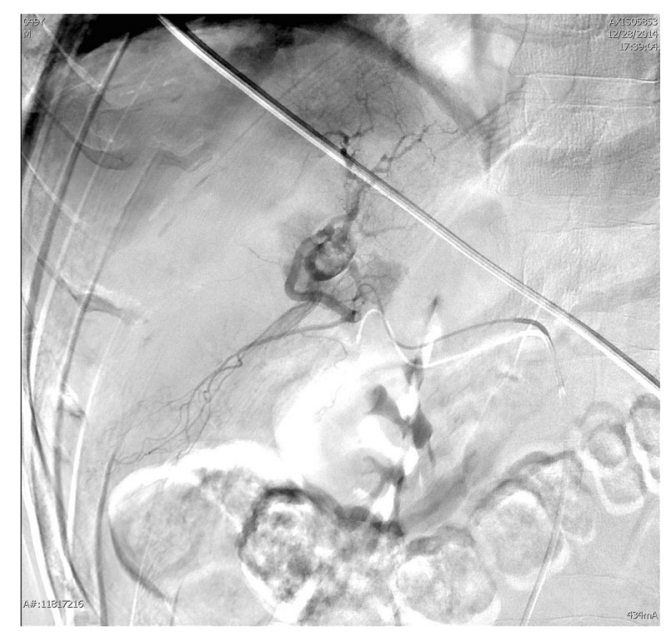

Fig. 2 The patient underwent diagnostic angiography demonstrating a large pseudoaneurysm 


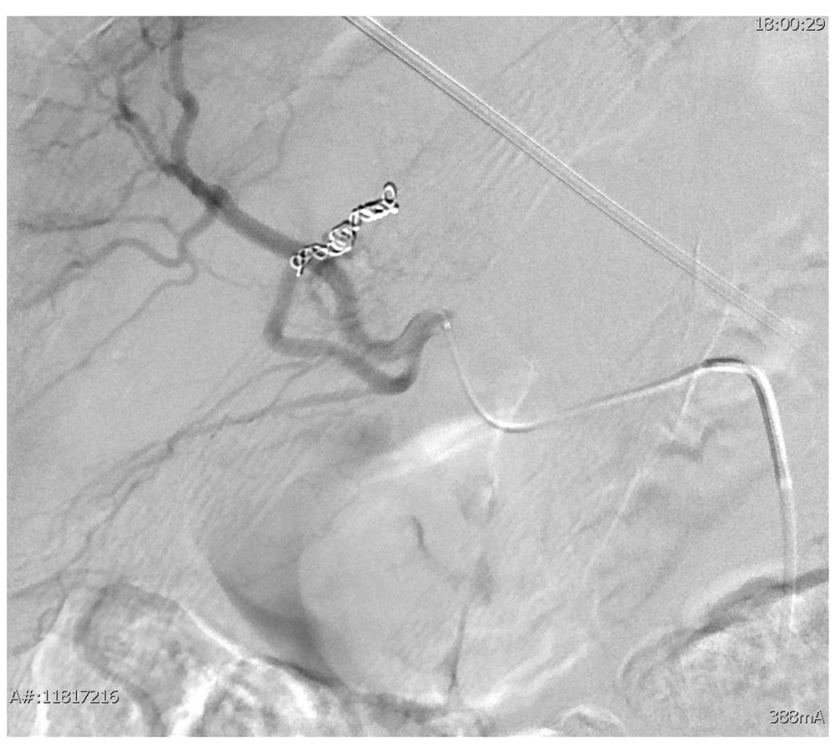

Fig. 3 The pseudoaneurysm was successfully treated with coil embolization

liver [22]. The series included both blunt and penetrating trauma. Presumably, this is more common when embolization is used as part of damage control. The patient is tightly packed, which may impede portal venous flow. The injury itself will disrupt some of the arterial flow, as well as potentially major branches of the portal vein. Angiographic embolization then disrupts arterial blood flow, making hepatic necrosis almost inevitable.

The use of angiographic embolization for vascular injury identified within the liver in patients who are stable is somewhat controversial. The natural history of these asymptomatic pseudoaneurysms in the spleen is well known where $70 \%$ go on to become symptomatic without embolization [23]. However, the same may not be true in the liver. In a recent article by Velmahos' group at Massachusetts General Hospital, following blunt trauma, small pseudoaneurysms were successfully observed [24•]. As the risk of hepatic necrosis following
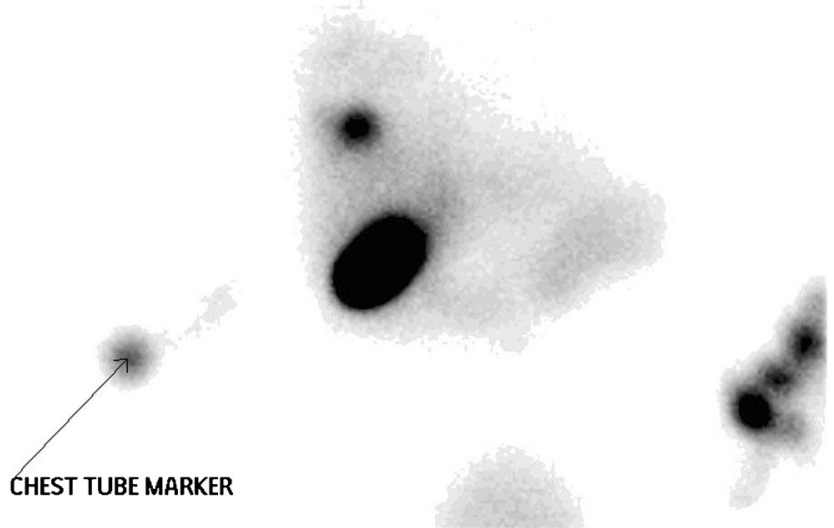

Fig. 4 Nuclear medicine biliary scan demonstrates a leak up through the diaphragmatic injury. This can be seen as contrast accumulating in the chest tube

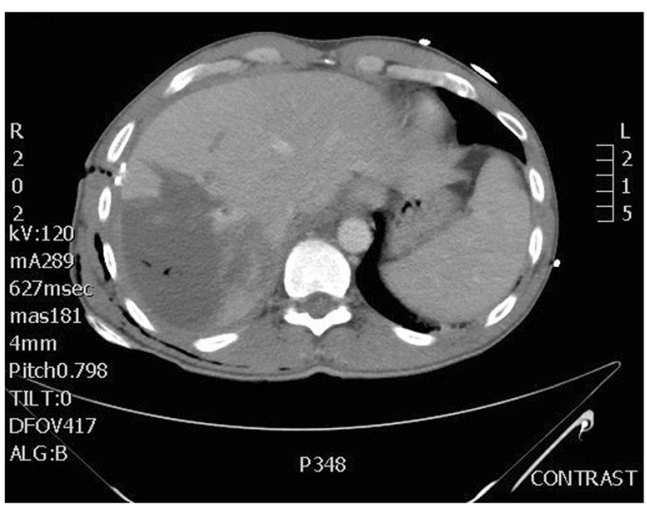

Fig. 5 Penetrating injury to the right lobe of the liver treated with embolization. The patient developed major hepatic necrosis following his embolization

angiographic embolization of the liver is so high, my general practice is to try to avoid hepatic embolization in asymptomatic patients. If the pseudoaneurysms is very large, we try to employ as selective an embolization strategy as possible. Occlusion with coils is preferable to widespread use of gel foam, as we believe that the incidence of hepatic necrosis with selective embolization is less when a more precise embolization strategy is used.

If major hepatic necrosis does occur, our preferred strategy is early hepatic lobectomy [25] (Figs. 5 and 6). We have demonstrated that early hepatic lobectomy is safe and likely more efficacious than repeated attempts at debridement or placement of drains in the interventional radiology suite.

\section{Adjuncts for Hemostasis}

Surgical therapy with angiographic embolization as needed is the therapy most widely selected for treating liver injuries. Hemorrhage can be supplemented with a number of other strategies. Hemostatic bandages have a great role in helping to arrest hepatic hemorrhage. Combat Gauze is likely the most commonly used agent but requires removal, as it is not

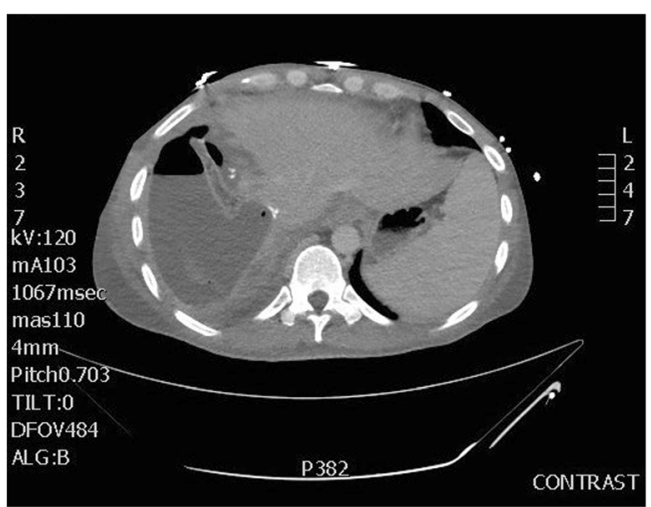

Fig. 6 Post right hepatic lobectomy collection of bile. This occurred despite drains being placed in the operating room. This was successfully treated with percutaneous drainage 
biodegradable. Recently, a nano-engineered hemostatic agent has been described, the Rapid Trauma Hemostat [26]. This has been demonstrated to be effective in a number of models of hemorrhage, including that of liver injury.

Short electrical pulses have been demonstrated to be effective in a rabbit liver injury model [27]. Electrical pulsation constrict blood vessels and can be hemostatic. Animals treated with electrical current had far superior hemostasis than those treated with mechanical pressure or those not treated at all. Liver temperature did rise in the animals, but the maximum liver temperature was $39.4^{\circ} \mathrm{C}$.

Arginine vasopressin has been studied in a swine model of penetrating liver injury that involved approximately $40 \%$ loss of estimated blood volume [28]. The animal was treated with vasopressin and had higher blood pressures, and lower hemorrhage volume, when compared to animals treated with either epinephrine or saline. In addition, blood flow to the liver, GI track, and kidney returned to normal more quickly in the animals treated with vasopressin. Finally, fibrogen gamma chain liposomes have been studied in a thrombocytopenic rat model of non-compressible liver injury [29]. Liposomes are thought to accumulate at the site of bleeding via interaction with activated platelets and then augment platelet aggregation. In this liver model, therapy was demonstrated to be safe and effective controlling hemorrhage.

\section{Liver Support}

Hepatic failure is relatively infrequent in patients with significant liver injury, despite the fact that right hepatic lobectomy is sometimes required with a significant loss of functioning hepatic parenchyma. The liver that remains seems to be sufficient to support hepatic function in patients. We recently cared for two patients, one with penetrating trauma and one with blunt trauma that presented in severe hemorrhagic shock and required hepatic resection for hemostasis. Both of these patients developed profound hepatic failure postoperatively. They were supported with molecular absorbent recirculating system (MARS). Both recovered and survived.

The MARS is currently the most extensively used liver support system, having been in use since 1996 [30-32]. The system functions on dialysis, filtration, and absorption. Blood is exposed to a biocompatible high flux albumin dialysis filter with removal of both water soluble and albumin-bound toxins $[33,34]$. Twenty percent albumin dialysate is run counter current to the blood to transfer albumin-bound toxins to the dialysate in a system very similar to continuous renal replacement therapy [35]. The exogenous albumin dialysate is then "regenerated" by dialysis against a conventional dialysate bath and subsequent absorption from charcoal and exhange resin colums [33]. The regenerated albumin dialysate is then re-circulated to repeat the process. The usual time of treatment is 6 to $8 \mathrm{~h}$ per day [30].

\section{Conclusion}

Penetrating injuries are likely decreasing. In those with penetrating injury, however, liver injuries are common. Selective non-operative management is safe but patients must be closely monitored as some will fail. The principles of operative management have not changed though some new technologies are becoming available. Angiographic embolization can be lifesaving but is also morbid. Minimally invasive approaches are likely to increase in use. In a small group of patients, advanced hepatic support may be needed.

\section{Compliance with Ethics Guidelines}

Conflict of Interest Thomas M. Scalea declares no conflict of interest.

Human and Animal Rights and Informed Consent This article does not contain any studies with human or animal subjects performed by any of the authors.

\section{References}

Papers of particular interest, published recently, have been highlighted as:

- Of importance

•- Of major importance

1. Zago TM, Pereira BM, Nascimento B, Alves MSC, Rodrigues T, Calderan A, et al. Hepatic trauma: a 21-year experience. Rev Col Bras Cir. 2013;40(4):318-22.

2. Jacobs LM. Development and evaluation of the advanced trauma operative management course. J Trauma Acute Care Surg. 2003;55(3):471-9.

3. Moore EE. Mandatory laparotomy for gunshot wounds penetrating the abdomen. Am J Surg. 1980;140(6):847-51.

4.• Como JJ, Bokhari F, Chiu WC, Duane TM, Holevar MR, Tandoh MA, et al. Practice management guidelines for selective nonoperative management of penetrating abdominal trauma. J Trauma. 2010;68(3):721-33. This is an EAST evidence review of indications for nonoeprative management of penetrating trauma. It codifies the best evidence available in details and indications for nonoperative management of penetrating torso trauma.

5. Phillips T, Sclafani SJ, Goldstein A, Scalea T, Panetta T, Shaftan G. Use of the contrast-enchanced CT enema in the management of penetrating trauma to the flank and back. J Trauma. 1986;26:593601.

6. Demetriades D, Rabinowitz B, Sofianos C. Non-operative management of penetrating liver injuries: a prospective study. Br J Surg. 1986;73:736-7.

7. Chmielewski GW, Nicholas JM, Dulchavsky SA, Diebel LN. Nonoperative management of gunshot wounds of the abdomen. Am Surg. 1995;61:665-8. 
8. Demetriades D, Gomez H, Chahwan S, et al. Gunshot injuries to the liver: the role of selective nonoperative management. J Am Coll Surg. 1999;188:343-8.

9. Renz BM, Feliciano DV. Gunshot wounds of the right thoracoabdomen: a prospective study of nonoperative management. J Trauma. 1994;37:737-44.

10. Demetriades D, Hadjizacharia P, Constantinou C, et al. Selective nonoperative management of penetrating abdominal solid organ injuries. Ann Surg. 2006;244:620-8.

11. Shanmuganathan K, Mirvis SE, Chiu WC, Killeen KL, Hogan GJ, Scalea TM. Penetrating torso trauma: triple-contrast helical CT in peritoneal violation and organ injury - a prospective study in 200 patients. Radiology. 2004;231:775-84.

12. Sorour MA. Conservative approach in the management of isolated penetrating liver trauma. Alex J Med. 2013;49(2):125-32.

13. Starling SV, de Rodrigues BL, Martins MPR, Silva MSA, Drumond DAF. Nonoperative management of gunshot wounds on the right thoracoabdomen. Rev Col Bras Cir. 2012;39(4):286-94.

14. Navsaria PH, Nicol AJ, Krige JE, Edu S. Selective nonoperative management of liver gunshot injuries. Ann Surg. 2009;249(4):6536.

15. Feliciano DV, Jordan GL, Bitondo CG, Mattox K, Burch JM, Cruse PA. Management of 1000 consecutive cases of hepatic trauma (1979-1984). Am Surg. 1986;204(4):438-43.

16.• Ordonez CA, Parra MW, Salamea JC, Puyana JC, Millan M, Badiel $\mathrm{M}$, et al. A comprehensive five-step surgical management approach to penetrating liver injuries that require complex repair. J Trauma Acute Care Surg. 2013;75(2):207-11. This article is written by a very busy group in Cali, Colombia. It details a step by step approach to penetrating liver injury. The article stresses surgical techniques for the most complicated injuries.

17. Poggetti RS, Moore EE. Balloon tamponade for bilobar transfixing hepatic gunshot wounds. J Trauma Acute Care Surg. 1992;33(5): 694-7.

18. Fraga GP, Zago TM, Pereira BM, Calderan TRA, Silveira HJV. Use of Sengstaken-Blakemore intrahepatic balloon: an alternative for liver-penetrating injuries. World J Surg. 2012;36:2119-24. This manuscript is the largest series of the use of Sengstakenblakemore tube. The series reports very good results.

19. Becker A, Mizrahi H, Chulsky A. Balloon tamponade with Sengstaken-Blakemore tube for penetrating "core" liver injury. J Curr Surg. 2013;3(1):47-8.

20. Beitner MM, Suh N, Dowling R, Miller JA. Penetrating liver injury managed with a combination of balloon tamponade and venous stenting. A case report and literature review. Injury Int J Care Injure. 2012;43:119-22.

21. O’Malley E, Boyle E, O'Callaghan A, Coffey JC, Walsh SR. Role of laparoscopy in penetrating abdominal trauma: a systematic review. World J Surg. 2013;37:113-22.
22. Dabbs DN, Stein DM, Scalea TM. Major hepatic necrosis: a common complication after angioembolization for treatment of highgrade liver injuries. J Trauma Acute Care Surg. 2009;66(3):621-9.

23. Davis KA, Fabian TC, Croce M. Improved success in nonoperative management of blunt splenic injuries: embolization of splenic artery pseudoaneurysms. J Trauma Acute Care Surg. 1998;44(6):100815.

24. Michailidou M, Velmahos GC, Wilden G, Alam HB, de Moya M, Chang Y. Blush on trauma computed tomography: not as bad as we think. J Trauma Acute Care Surg. 2012;73(3):580-6. This manuscript clearly demonstrated that certain small contrast blushes seen on high resoulution CT scanning of the abdomen can be safely observed. The article suggests that size of blush, AIS of the abdomen and hemodynamics predicts successful resolution.

25. Dabbs DN, Stein DM, Philosophe B, Scalea TM. Treatment of major hepatic venous: lobectomy versus serial debridements. J Trauma. 2010;69:562-67.

26. Arnaud F, Okada T, Solomon D, Haque A, Carroll EE, Sagini E, et al. Initial evaluation of a nano-engineered hemostatic agent in a severe vascular and organ hemorrhage swine model. J Trauma Acute Care Surg. 2012;73(5):1180-7.

27. Mandel Y, Malki G, Adawi E, Glassberg E, Afek A, Zagetzki M, et al. Hemorrhage control of liver injury by short electrical pulses. PLoS ONE. 2013;8(1):1-7.

28. Voelckel WG, Raedler C, Wenzel V, Lindner KH, Krismer AC, Schmittinger CA, et al. Arginine vasopressin, but not epinephrine, improves survival in uncontrolled hemorrhagic shock after liver trauma in pigs. Crit Care Med. 2003;31(4):1160-5.

29. Nishikawa K, Hagisawa K, Kinoshita M, Shono S, Katsuno S, Doi $\mathrm{M}$, et al. Fibrinogen $\mathrm{y}$-chain peptide-coated, ADPencapsulated liposomes rescue thrombocytopenic rabbits from noncompressible liver hemorrhage. J Thromb Haemost. 2012;10:2137-48.

30. Podoll AS, DeGolovine A, Finkel KW. Liver support systems - a review. ASAIO J. 2012;58:443-9.

31. Liu JP, Gluud LL, Als-Nielsen B, Gluud C. "Artificial and bioartificial support systems for liver failure." Cochrane Database Syst Rev. 2004;(1)CD003628.

32. Mitzner SR, Kaplan AA. Liver support 2009: time for another revolution? Ther Apher Dial. 2009;13:381-3.

33. Inderbitzin D, Muggli B, Ringger A, et al. Molecular absorbent recirculating system for the treatment of acute liver failure in surgical patients. J Gastrointest Surg. 2005;9:1155-61. discussion 11611162.

34. Jalan R, Sen S, Steiner C, Kapoor D, Alisa A, Williams R. Extracorporeal liver support with molecular adsorbents recirculating system in patients with severe acute alcoholic hepatitis. Hepatology. 2003;38:24-31.

35. Patzer 2nd JF, Bane SE. Bound solute dialysis. ASAIO J. 2003;49: 271-81. 